\title{
Influence of the Gyroscopic Effects on Friction Induced Vibration in Aircraft Braking in System
}

\author{
Gabriel Hurel ${ }^{1,2}$, Jean-Frédéric Diebold ${ }^{1}$, \\ Sébastien Besset ${ }^{2}$, Louis Jézéquel ${ }^{2}$ \\ ${ }^{1}$ Messier-Bugatti-Dowty \\ ${ }^{2}$ Ecole Centrale de Lyon \\ Published on September 17, 2012 \\ doi:10.4271/2012-01-1804
}

\begin{abstract}
Aeronautical brakes are subject to non-linear unstable vibrations. In particular, two modes appear and present a risk for the structure. Firstly, the whirl modes consist of a rotating bending motion of the axle out-of-phase with the brake torque tube. It is due to a coupling of two bending modes of the axle in orthogonal directions. Secondly, the brake squeal mode resulting from stick-slip or sprag-slip phenomena consists of a rotational motion of the brake around the axle. Those vibrations are not resulting from an external excitation but are friction-induced self-excited. Hence, they are dependent on tribological phenomena specific to carbon disks and are in particular controlled by the friction coefficient $\mu$.

In order to take into account the dynamical aspect in brake design, Messier-BugattiDowty wants to simulate modes and acceleration g's levels. This article deals with the improvement of such a model.

A finite element of the brake exists. It is able to reproduce whirl modes and squeal mode. In order to improve it, physical phenomena must be introduced. Here, the impact of gyroscopic effects is evaluated. For this, an analytical model is built to determine the consequences on frequencies and stability.
\end{abstract}

\section{Introduction}

Friction induced vibrations are present in many applications. Squeaky doors and violins are examples in which noise is created from vibrations due to two interfaces contact characterized with a friction law. In railway and automotive industries, these phenomena are studied for braking systems [1, 2]. Indeed, significant energy is involved and vibrations produce important noise and present a risk for the brake structure.

In the aeronautical industry, the objective is not yet to reduce noise but to avoid premature worn and broken equipment. Many tests are performed to check that the brakes do not vibrate, but they are long and expensive. Models are used to predict the brakes' dynamic performance and to understand dynamic phenomena observed during tests, in order to improve design.

Contrary to automotive brakes, aircraft brakes are axisymmetric. A stack of carbon disks, called heat sink, is placed between pistons and torque tube (see Figure 1). Stators secured to the axle and rotors secured to the wheel are arranged alternatively. During the braking phase, a hydraulic pressure is applied on the carbon disks through pistons. This compressive force generates a friction effort between rotors and stators over the entire circumference. It 
results in a torque, opposed to the wheel rotation. The brake rod transmits the torque to the attach lug linked to the landing gear. The mechanical energy is changed into thermal energy through friction and absorbed by the heat sink [3].
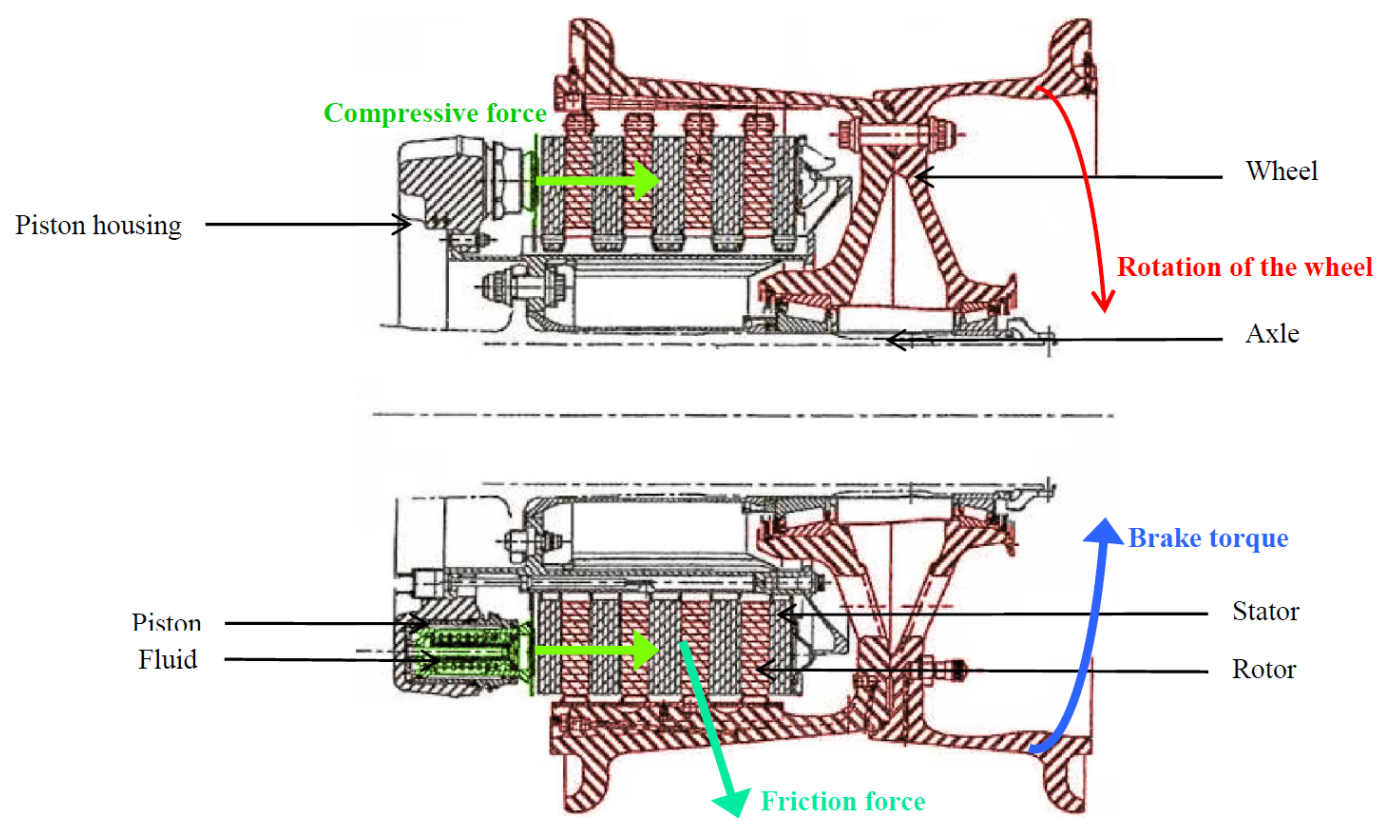

Figure 1: Sectional view of an aircraft brake system [3].

Many braking stops are performed on a test frame. They correspond to landing or taxiing. The brake is attached by the axle, the brake rod and the attach lug. This mechanism is subject to unstable vibrations during the braking. Frequency range is $50-2000 \mathrm{~Hz}$. During a braking, several different modes may appear. Their levels increase rapidly to reach a stationary limit cycle. Figure 2 shows an example of a braking with appearance of three different modes.

Two types of brake induced vibration may be observed in the low frequency range. The rotation of the brake about the axle called squeal (Figure 3a) results from stick-slip or spragslip phenomena. Unlike stick-slip, sprag-slip [4] helps to explain vibrations with a constant friction coefficient and a buttressing of the brake rod. In the first phase, the brake torque induces a flexion of the rod. This energy is relaxed by slipping on the friction interface.

The spin motion of the axle is called whirl (Figure 3b). This complex mode results from the coupling of two bending modes of the axle $[5,6]$. This coupling depends on the carbon friction coefficient. Several modes of whirl exist depending on order of the mode of the axle.

In order to predict apparition and level of vibrations, a model is needed. A finite element model was built to understand the brake dynamic behaviour [7]. It is able to reproduce the squeal and whirl modes. This model is currently used to identify modes of the structure and perform stability studies. 

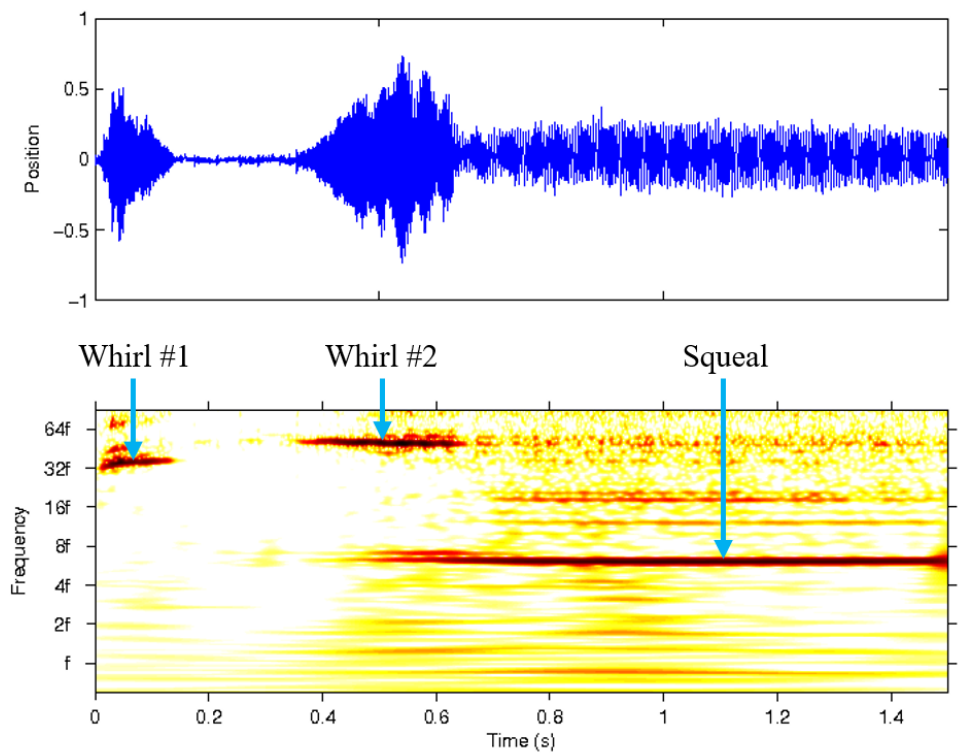

Figure 2: Example of vibrations during braking with frequency analysis by wavelet.

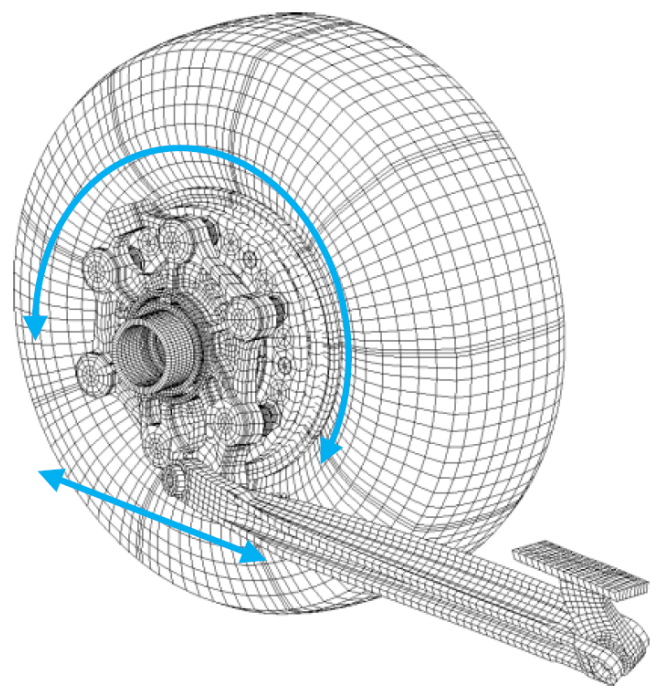

(a)

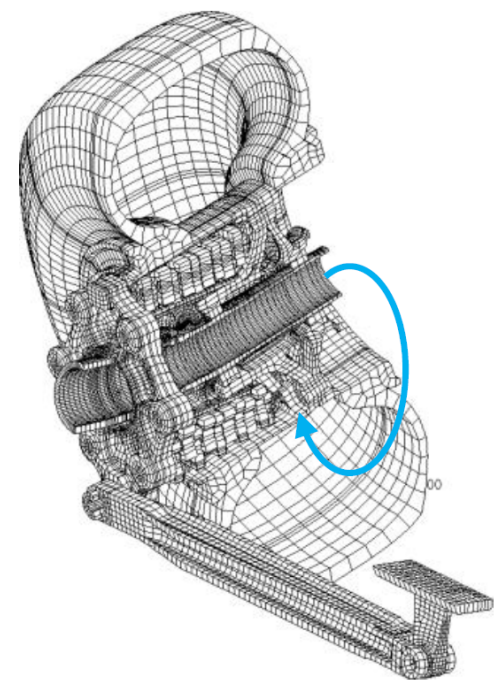

(b)

Figure 3: Two modes of vibration on aircraft brake system ((a): squeal mode, (b): whirl mode). 


\section{Finite element model description}

This finite element model corresponding to the test configuration is built from CAD. Figure 4 shows the meshed brake system. The frequency range requires parts from the landing gear: axle, brake rod and attach lug.

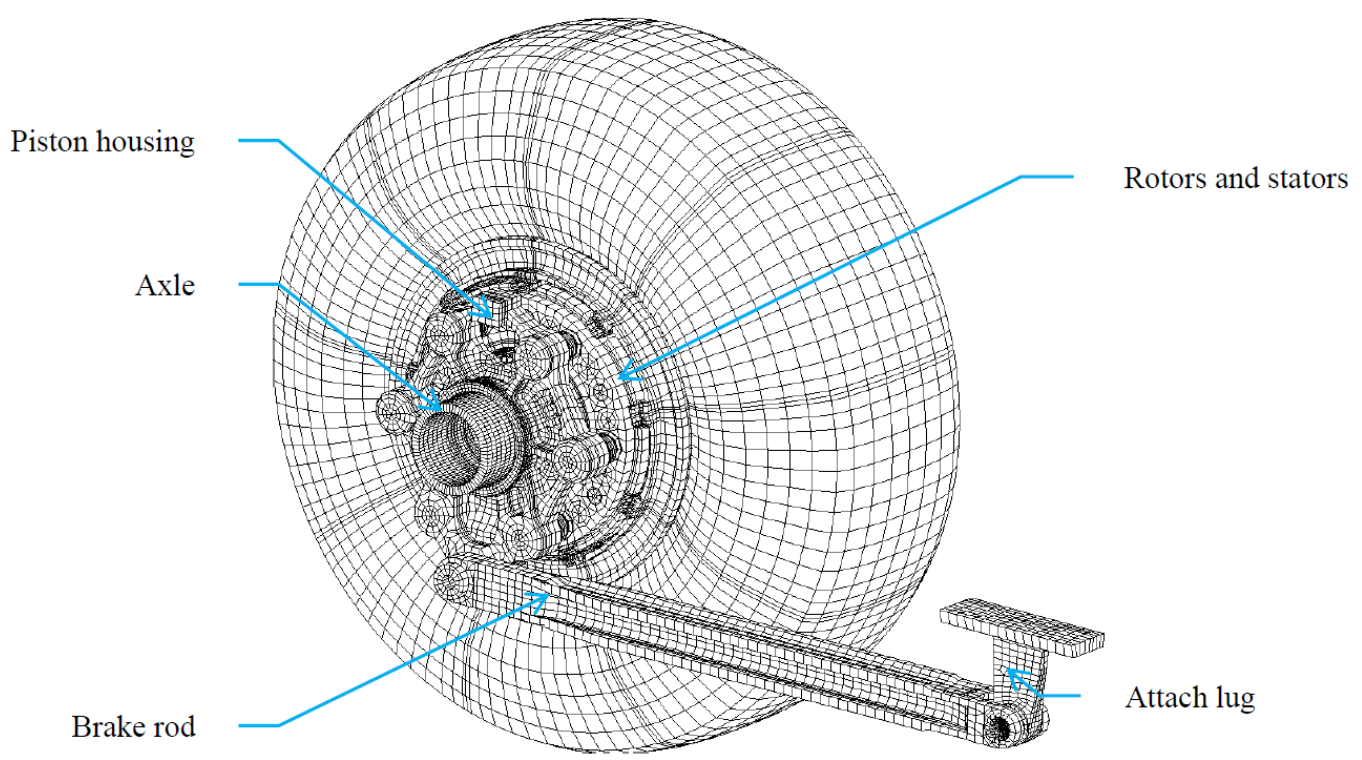

Figure 4: Finite element model of brake system.

\subsection{Friction Modeling}

For now, the value of rotation speed is not important here because the friction is saturated. The Coulomb friction law is modelled at disks interface. Contact stiffness $k_{c}$ is introduced to prevent permeation of disks and to retrieve compressive force $N$. A tangential force $T$ proportional to the normal force and friction coefficient $\mu$ is generated (figure 5). This way, the friction coefficient $\mu$ is constant on all the disks' surface but the friction force is located where pressure is important [8]:

$$
T=\mu N=\mu k_{c}\left(z_{A}-z_{B}\right)
$$




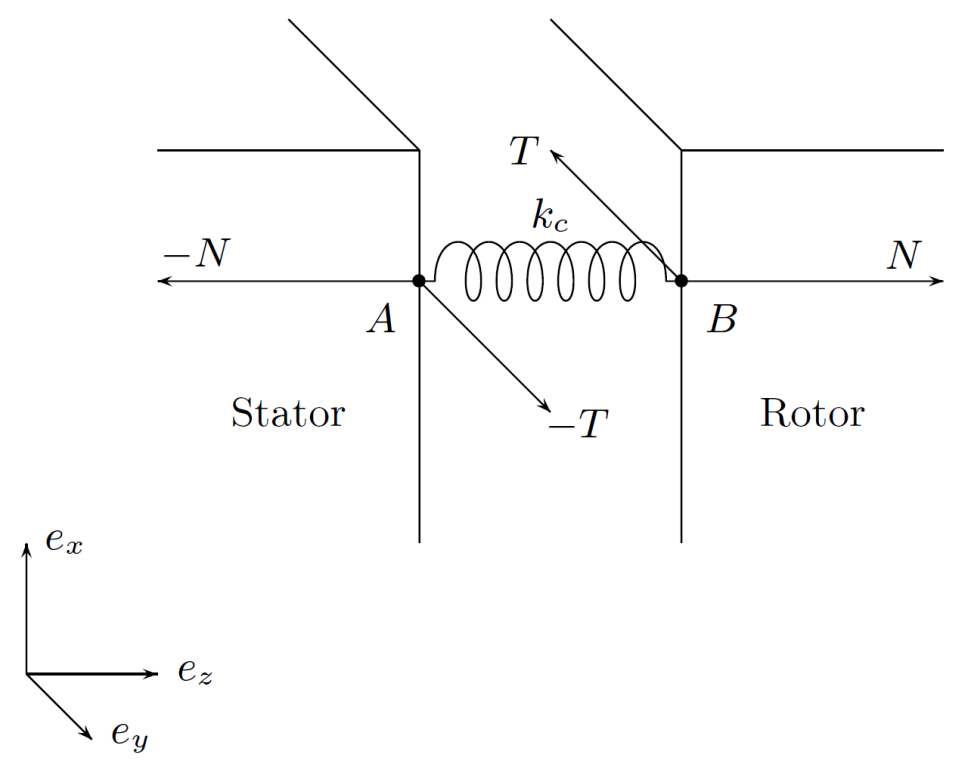

Figure 5: Friction modelling at the disks rubbing interface between two nodes $A$ and $B$.

\section{Modal and stability analysis}

\subsection{Computation Technique}

The introduction of friction adds to the symmetric stiffness matrix $\mathbf{K}_{S}$ an asymmetric ma$\operatorname{trix} \mu \mathbf{K}_{F}$.

$$
\mathbf{K}=\mathbf{K}_{S}+\mu \mathbf{K}_{F}
$$

The system is no longer conservative and energy is brought to the structure from friction. In this case, unstable modes may appear. Actually, dynamic equation is written with mass matrix $\mathbf{M}$, damp matrix $\mathbf{C}$ and stiffness matrix $\mathbf{K}$ :

$$
\mathbf{M} \ddot{X}+\mathbf{C} \dot{X}+\mathbf{K} X=0
$$

Calculating modes $\left(\lambda_{i}, \varphi_{i}\right)$ is equivalent to solve matrix equation

$$
\left(\lambda_{i}^{2} \mathbf{M}+\lambda_{i} \mathbf{C}+\mathbf{K}\right) \varphi_{i}=0
$$

On a conservative system, eigenvalues $\lambda_{i}$ are purely imaginary. This is not the case here if $\mu \neq 0$ because stiffness matrix $\mathbf{K}$ is not symmetric. So eigenvalues $\lambda_{i}$, are either purely imaginary or complex. In this case, they may be written $\lambda=a+\boldsymbol{i} \omega$ where $\omega$ is the pulsation of the mode related with the frequency $f=2 \pi \omega$. Parameter $a$ is a modal damping coefficient which determines stability: if $a$ is negative or zero, the system is stable but if a is positive the system is unstable. These two coefficients $a$ and $\omega$ are dependent on friction coefficient $\mu$. A stability analysis can be performed by studying the frequency variation against friction coefficient. An example is given in figure 6 .

\subsection{Results}

The finite element model is able to reproduce modes observed during braking test in the low frequency range. The whirl mode appears from a value of the friction coefficient $\mu$. Even if there is a coupling, the model requires an update to retrieve the correct whirl mode. 


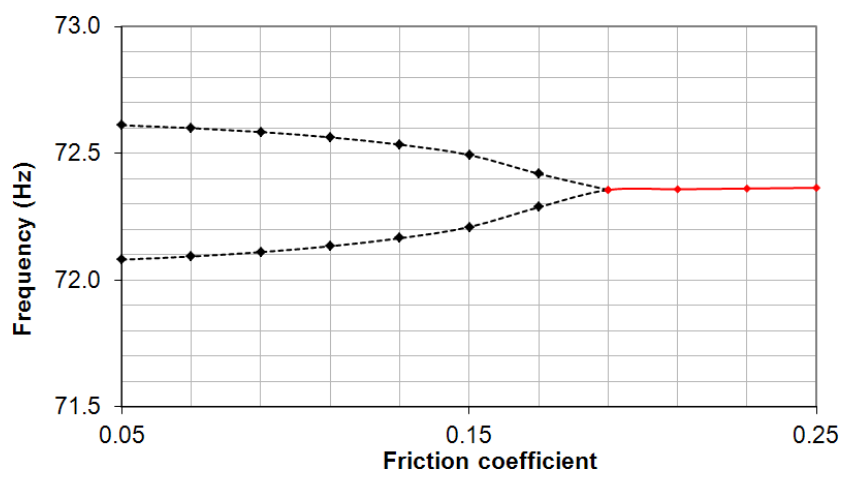

(a)

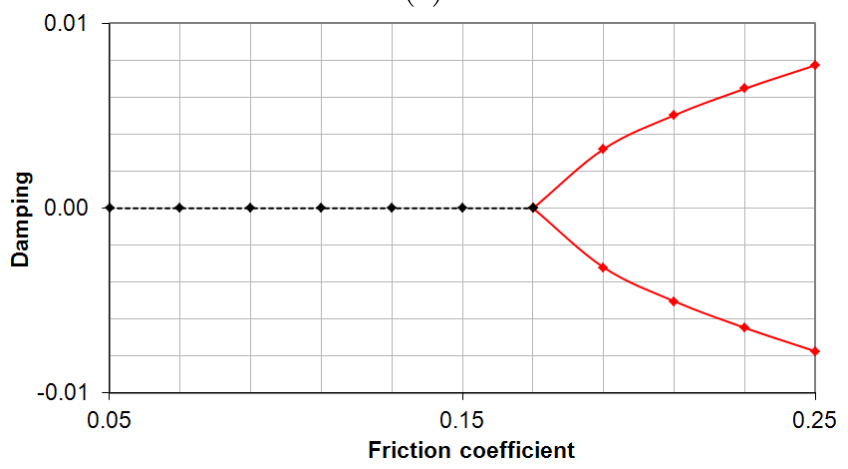

(b)

Figure 6: Stability analysis for the first whirl ((a): frequency, (b): damping).

\section{Improvement of the dynamic model}

Different dynamic behaviours of the brake structure are observed for landing stops or taxiing stops during tests. Especially, the whirl mode appears more often during landing stops than taxiing stops. Moreover, the finite element model underestimates the frequency of this mode. The objective of this study is to identify the link between the value of the speed of rotation of the wheel, which is significantly higher in landing than in taxiing stops, and the characteristics of the whirl mode: frequency and stability indicators.

Gyroscopic effects could be the root cause of these observations. In order to determine whether these phenomena should be introduced in the finite element model, a study on a simple analytical model is conducted. First, influence of gyroscopic effects without braking on the whirl mode's frequency is evaluated. Then, friction is introduced to quantify its impact on stability for the whirl mode.

\subsection{Impact of Gyroscopic Effect on Modes Frequencies}

Gyroscopic effects are studied on aircrafts engines, where the rotation speed of the rotors is very high. The wheel and brake system may also be subject to gyroscopic effects. Indeed, when landing the wheel rotates with an important speed, especially at the beginning of braking. In addition, the load of the aircraft causes a flexion of the axle perpendicular to the wheel rotation. Both cause a gyroscopic force in the third direction. This study is done to find out if these effects must be injected into the finite element model.

To quantify these effects, a simple model is used (figure 7) with equations used for rotor dynamics [9]: an axisymmetric cantilever beam free with constant section represents the axle. Rotating parts are considered as a flywheel. This flywheel is represented by its mass is $M$ and its moments of inertia about $\vec{e}_{x}$ and $\vec{e}_{y}, I_{1}$ and about $\vec{e}_{z}, I_{3}$. Movements of the 
beam are allowed in two directions: $u$ and $v$ are the displacements along respectively $\vec{e}_{x}$ and $\vec{e}_{y}$ axis. $\theta$ and $\psi$ are angles of rotation of the wheel around respectively $\vec{e}_{x}$ and $\vec{e}_{y}$ axis. A rotation speed is imposed to the flywheel at pulsation $\Omega$.

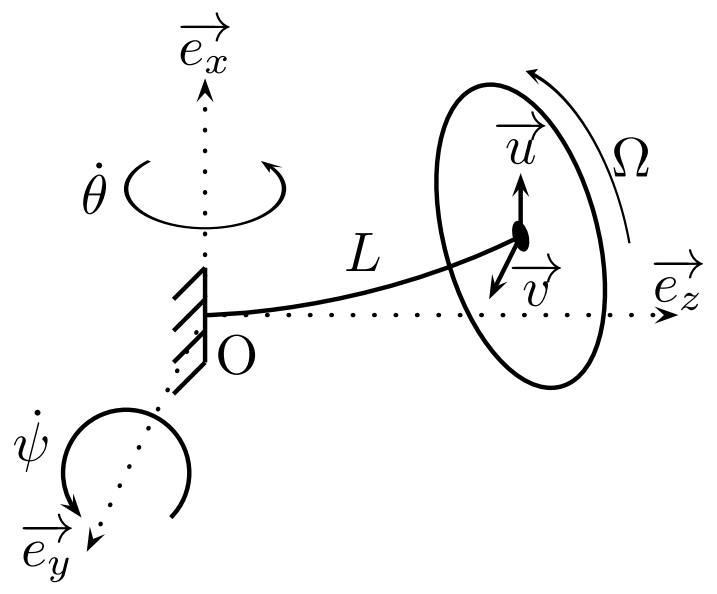

Figure 7: Simple model to study gyroscopic effects on wheel and brake system.

The axle is considered as a massless body. Its modal shapes are given by the functions $f_{x}(z)$ and $f_{y}(z)$. The structure can be reduced to two DOF $q_{1}$ and $q_{2}$ :

$$
\begin{aligned}
u(z) & =f_{x}(z) q_{1} \\
v(z) & =f_{y}(z) q_{2} \\
\psi & =\frac{\mathrm{d} f_{x}}{\mathrm{~d} z}(L) q_{1} \\
\theta & =\frac{\mathrm{d} f_{y}}{\mathrm{~d} z}(L) q_{2}
\end{aligned}
$$

By applying the Lagrange equations, the following system is obtained [9]:

$$
\left[\begin{array}{cc}
m & 0 \\
0 & m
\end{array}\right]\left(\begin{array}{l}
\ddot{q}_{1} \\
\ddot{q}_{2}
\end{array}\right)+\Omega\left[\begin{array}{cc}
0 & -g \\
g & 0
\end{array}\right]\left(\begin{array}{l}
\dot{q}_{1} \\
\dot{q}_{2}
\end{array}\right)+\left[\begin{array}{ll}
k & 0 \\
0 & k
\end{array}\right]\left(\begin{array}{l}
q_{1} \\
q_{2}
\end{array}\right)=\mathbf{0}
$$

where $m=M+\frac{4 I_{1}}{L^{2}}, g=\frac{8\left(I_{3}-I_{1}\right)}{L^{2}}$ and $k=\frac{4 E I_{f}}{L^{3}}$. Resolution of the equation 9 gives two solutions corresponding to two pulsation frequencies:

$$
\begin{aligned}
& r_{1}=i \omega_{1}=i \sqrt{\omega_{0}^{2}+\frac{g^{2} \Omega^{2}}{2 m^{2}}\left(1-\sqrt{1+\frac{4 m^{2} \omega_{0}^{2}}{g^{2} \Omega^{2}}}\right)} \\
& r_{2}=i \omega_{2}=i \sqrt{\omega_{0}^{2}+\frac{g^{2} \Omega^{2}}{2 m^{2}}\left(1+\sqrt{1+\frac{4 m^{2} \omega_{0}^{2}}{g^{2} \Omega^{2}}}\right)}
\end{aligned}
$$

The pulsation when the wheel is not rotating is $\omega_{0}=\sqrt{k / m}$. It is interesting to note the appearance of a coupling between both bending modes. However, there is no modal damping and no preferred mode. The mode shapes are the same as the whirl mode. The second frequency $\omega_{2}$ corresponds to the case where the vibration mode rotates in the same way as the wheel rotation $\Omega$, it is a direct precession. Conversely, the first frequency $\omega_{1}$ corresponds to the case where both rotations are in opposite directions, it is reverse precession. 
In figure 8, a Campbell diagram shows the whirl modes frequencies against the wheel rotation speed $\Omega$. The maximum speed of the aircraft is reached during landing. At this moment, the impact on frequency of the whirl mode is between 2 and $4 \%$.

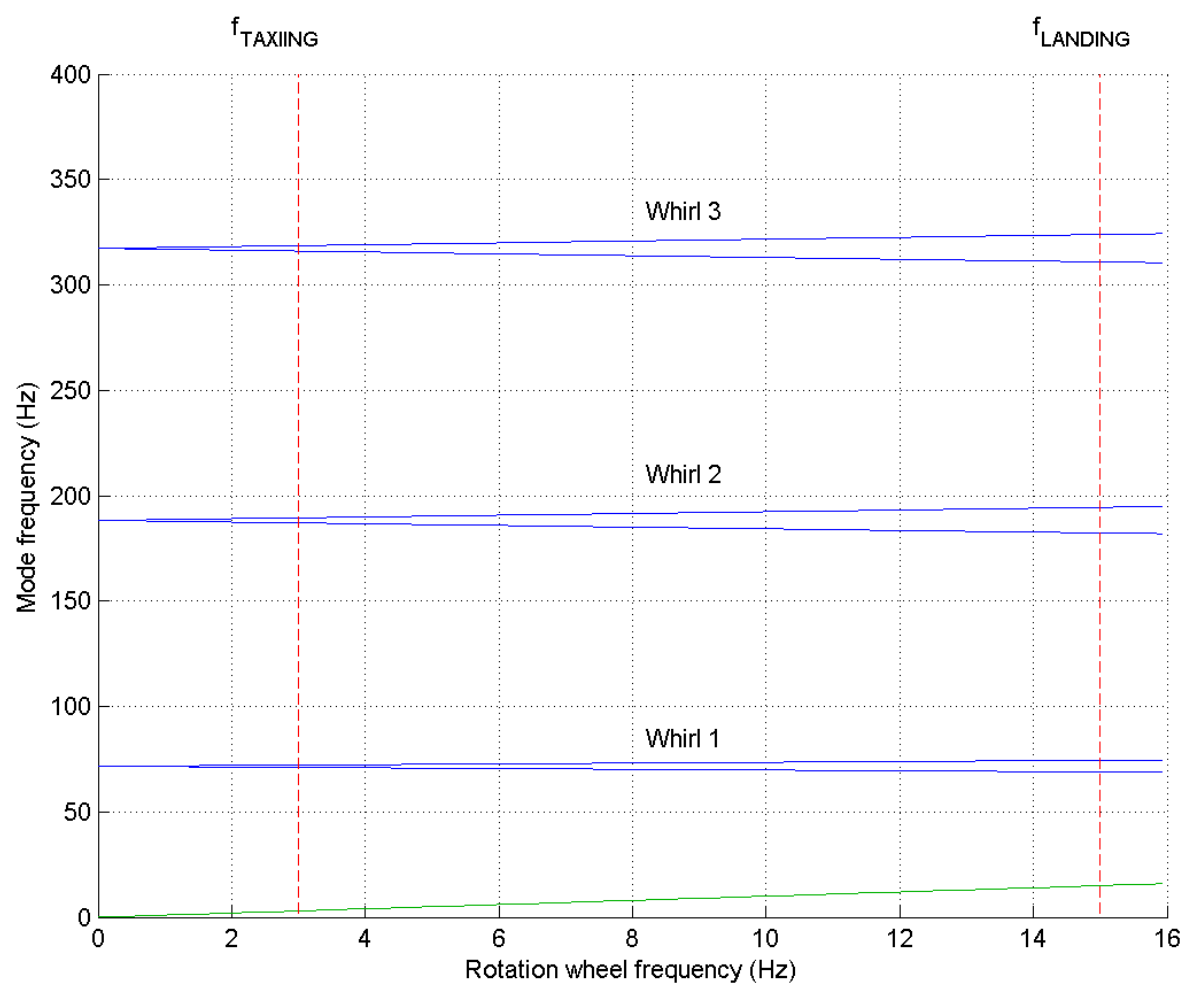

Figure 8: Campbell diagram with frequencies of the first whirl modes.

\section{$4.2 \quad$ Impact of gyroscopic effects on stability}

To evaluate the impact of gyroscopic effects on stability [10], a Coulomb friction law is introduced in the simple model between a rotor secured to the wheel and a fixed stator. When the wheel rotates in $\psi$, on the one hand, an elastic force reacts on the wheel in $\psi$ and on the other hand the friction creates a resultant force in $\vec{e}_{y}$ due to inhomogeneous pressure on the disks. This force bends the beam and rotates the wheel in $\theta$. The system equation becomes:

$$
\left[\begin{array}{cc}
m & 0 \\
0 & m
\end{array}\right]\left(\begin{array}{l}
\ddot{q}_{1} \\
\ddot{q}_{2}
\end{array}\right)+\Omega\left[\begin{array}{cc}
0 & -g \\
g & 0
\end{array}\right]\left(\begin{array}{l}
\dot{q}_{1} \\
\dot{q}_{2}
\end{array}\right)+\left[\begin{array}{cc}
k_{11} & -\mu k_{12} \\
\mu k_{12} & k_{11}
\end{array}\right]\left(\begin{array}{l}
q_{1} \\
q_{2}
\end{array}\right)=\mathbf{0}
$$

A bi-parametric study can be performed with friction coefficient, and speed rotation of the wheel $\Omega$. Figure 9 shows that the coupling of modes happens at $\mu=0$. The mode associated with positive real part eigenvalue is unstable for $\mu>0$. When $\Omega$ increases, the real parts decrease.

Hence, the gyroscopic decrease the modal damping. This results in a decrease of the growth rate of the whirl modes. However, in the range of the speed of rotation of the wheel, the difference of modal damping between landing and taxiing stops is negligible. Furthermore, the value of damping is not sufficient to explain appearance of instabilities.

Taking into account gyroscopic effects has improved correlation between the model and tests. The table 1 shows that only the whirl mode is concerned. The squeal mode is affected because it does not move rotating parts but parts linked to the landing gear. 


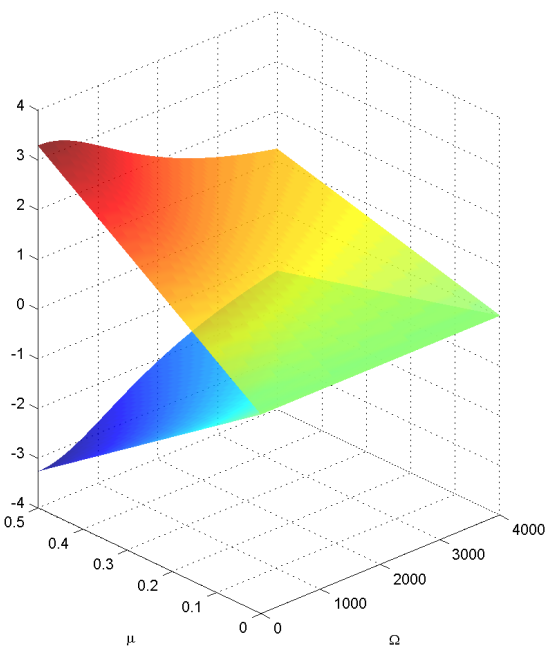

(a)

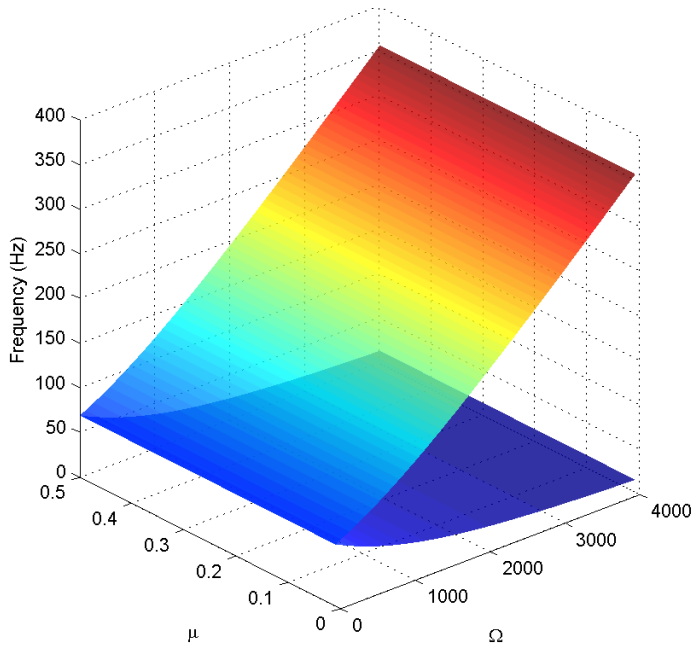

(b)

Figure 9: Whirl 1, (a): Real parts of eigenvalues versus $\mu$ and $\Omega$. (b): frequency versus $\mu$ and $\Omega$.

\begin{tabular}{lccc}
\hline Modes & Whirl 1 & Whirl 2 & Whirl 3 \\
\hline Difference on frequency & $3.8 \%$ & $3.1 \%$ & $2.0 \%$ \\
Difference on damping & $0.2 \%$ & $0.1 \%$ & $0.1 \%$ \\
\hline
\end{tabular}

Table 1: Improvement of the accuracy of frequencies and value of damping in the analytical model.

\section{Conclusion}

Messier-Bugatti-Dowty seeks to better understand the dynamic behaviour of brake systems in order to handle vibration levels. In order to better determine influence of the design, a finite element model is built. This model is able to reproduce complex modes observed through tests such as squeal and whirl modes thanks to insertion of friction terms.

However, before simulating its dynamic behaviour during braking, accuracy of the simulated modes frequencies can be further improved. In this paper, gyroscopic effects have been checked. The analytical model shows that taking into account gyroscopic effects can improve the finite element model. The accuracy of frequencies is improved especially for the whirl mode. To introduce these phenomena in the finite element model will be perform in a further work. On the other side, the impact of gyroscopic effects on stability is very low in the range of the speed of rotation of the wheel. They cannot explain the differences between landing and taxiing stops.

Other phenomena like pretension, the boundary conditions on the test frame, clearance in mechanical linkages and hydraulic coupling can considered to get a better representation of the dynamic behaviour of the brake. 


\section{References}

[1] N.M. Kinkaid, O.M. O'Reilly, and P. Papadopoulos. "Automotive disc brake squeal". Journal of Sound and Vibration 267.1 (2003), pp. 105-166. DOI: 10.1016 / S0022460X(02)01573-0 (cit. on p. 1).

[2] Xavier Lorang. "Instabilité des structures en contact frottant : Application au crissement des freins à disque de TGV". Theses. Ecole Polytechnique X, 2007 (cit. on p. 1).

[3] Jean Guichard. "Freinage à très hautes performances exemple aéronautique". Techniques de l'ingénieur Transmission de puissance mécanique : accouplement, embrayage, freinage base documentaire : TIB184DUO.ref. article : b5580 (1996) (cit. on p. 2).

[4] R. T. Spurr. "A Theory of Brake Squeal". Proceedings of the Institution of Mechanical Engineers: Automobile Division 15.1 (1961), pp. 33-52. DOI: 10.1243/PIME_AUTO_ 1961_000_009_02 (cit. on p. 2).

[5] Norbert Hoffmann, Michael Fischer, Ralph Allgaier, and Lothar Gaul. "A minimal model for studying properties of the mode-coupling type instability in friction induced oscillations". Mechanics Research Communications 29.4 (2002), pp. 197-205. DOI: 10. 1016/S0093-6413(02)00254-9 (cit. on p. 2).

[6] J.-J. Sinou, O. Dereure, G.-B. Mazet, F. Thouverez, and L. Jezequel. "Friction-induced vibration for an aircraft brake system-Part 1: Experimental approach and stability analysis". International Journal of Mechanical Sciences 48.5 (2006), pp. 536-554. DOI: 10.1016/j.ijmecsci.2005.12.002 (cit. on p. 2).

[7] F. Chevillot, J.-J. Sinou, and N. Hardouin. "Nonlinear transient vibrations and coexistences of multi-instabilities induced by friction in an aircraft braking system". Journal of Sound and Vibration 328.4-5 (2009), pp. 555-574. DOI: 10.1016/j.jsv.2009.08.028 (cit. on p. 2).

[8] Craig F. Chang and Thomas E. Ulbricht. "Asymmetric Approach in Solving Aircraft Brake Vibration". 2002. DOI: 10.4271/2002-01-2948 (cit. on p. 4).

[9] Michel Lalanne and Guy Ferraris. "Dynamique des rotors en flexion". Techniques de l'ingénieur Mécanique des éléments tournants base documentaire : TIB185DUO.ref. article : b5110 (1996) (cit. on pp. 6 sq.).

[10] B. Hervé, J.-J. Sinou, H. Mahé, and L. Jézéquel. "Analysis of squeal noise and mode coupling instabilities including damping and gyroscopic effects". European Journal of Mechanics - A/Solids 27.2 (2008), pp. 141-160. DOI: 10.1016/j.euromechsol.2007.05. 004 (cit. on p. 8).

\section{Contact information}

Gabriel HUREL

gabriel.hurel@ec-lyon.fr

Jean-Frederic DIEBOLD

jean-frederic.diebold@safranmbd.com

Sébastien BESSET

sebastien.besset@ec-lyon.fr 